\title{
LA COMPOSITION NIUSICALE ET LE RATIONNEL
}

Miclel Rigoni

(IRCAM)

"La musique est un exercice secret

d'arithunètique de l'esprit ignorant

qu'il compte"

G. W. LEIBNIZ

L'intitulé de cette communication vient paraphraser celui đu présent colloque.

Cela posé, il ne s'ngit pas pour autant de considérer la composition comme une réalité relevant uniquement du rationnel. Les lois de résonances acoustiques et l'activité compositionnelle sont deux mondes. Descartes avait lui-même atteint dans le Compendium Mhsicae les limites de l'explication rationaliste. Mais si l'on admet aisement la dimension irrationnelle dans l'oeuvre d'art, et la musique en particulier, la base mathématique du plénomène demeure.

Le développement teclınologique au vingtième siècle a, en outre, permis de scruter plus avant les vibrations du spectre sonore. L'analyse au microscope du spectre a révélé un monde qui a modifié la conception mème de la composition dans ces dernières décennies. L'évolution dans le domaine musical aura été d'autant plus spectaculaire. du dix-neuvième siècle à nos jours, que les romantiques étaient sans doute les plus éloignés possible de toutes spéculations scientifiques. Le propos est donc ici d'examiner à travers quelques éclats le rapport au rationnel cliez les compositeurs européens dans les deux derniers siecles.

L'origine pythagoricienne de la musique l'avait jadis amené à être associéc aux sciences et aux spéculations intellectuelles dans le Quadrivium.

Cette relation existe depuis l'origine, même si les approximations acoustiques des théories pythagoriciennes ont été relevées par Zarlino, Rameau et Descartes lui-mème.!

Beethoven a hérité de Haydn et Mozart un langage très rationalisé; mais ce n'est pas cet aspect qu'il va développer dans son an. Comme le souligne Célestin Deliège: la plrascologie beethovénienne, en ce qu'elle présente de plus abrupt, a engendré une nouvelle organisation du temps musical... C'est l'élément qui a fait pivoter toute la musique du classicisme au romantisme.

L'héritage que Wagner recevra est celui d'une musique de l'espril révolutionnaire. L'art $n$ 'est alors que métaphoriquement lié à la science.

La science, selon Wagner, aide à prendre conscience de la nécessité, tandis que l'art manifeste à l'homme cette nécessití.

Il convient de rappeler à ce propos que les romantiques ont été obsédés par le mythe de Faust qui finit par renoncer à trouver le sens de lexistence au travers de la science. Au début de sa Faust-Symphonie, Liszt mítaphorise la science avec une mílodie énigmatique de douze sons. Richard Strauss fera de mitme dans allso sprach Zarathustra. où il représente lit connaissance par un theme de douze sons qui sert de sujet à une fugue. La fugue demeure à cette épo-

I Miche!le BIGET, Le nombre pritexte, in Cahiors dh CIREM/N No Roten, 1986, p.22. 
que le moyen traditionnel pour un compositeur européen d'exprimer ce qui rèlève du savant. Strauss est en cela continuateur de Liszt.

Schönberg assignera aux douze sons chromatiques une toute autre mission.

Au tournant du dix-neuvieme et du vingtieme siècle un musicien comme RimskyKorsakov donne dans son Traité d'orchestration plus d'importance aux primat des lois acoustiques que Berlioz ne le faisait dans son propre traité, dont le discours porte la marque de son époque. C'est un signe des temps.

Dans les années 1900, l'écriture musicale voit le retour du nombre dans les procédures compositionnelles; ce nombre quelque peu oublié depuis Bach. La pensée thiosophique d'un Scriabine s'ome des proportions eurythmiques issues de la section d'or.

Si l'on en vient à Schönberg, le musicien du progrès, selon la définition d'Adorno, fait l'épreuve du passage à l'atonalité à travers la phase expressionniste. Les oeuvres sont pour la plupart sous-tendues par des textes poétiques ou dramatiques dans un univers des passions non maitriscies. L'exemple le plus hallucinant en est, évidemment, le monodrame Enwartung où la musique suit les émotions et les comportements de l'héroïne comıne un sismographe, pour reprendre l'expression adornienne. "La musique comme protocole de l'expression n'est plus expressive; elle est au-delà"...."Par son attitude protocolaire devant son objet, la musique devient elle-même objective. Ses explosions detruisent le rêve de la subjectivité non moins que les conventions." C'est une attitude radicale "à l'égard de la superstition de l'organique", poursuit Adorno "alors sa liquidation a dégagé une fois de plus l'idée d'oeuvre."2

Dans le Pierrot linaire, l'organique dans le langage est plus rationalisé, mais le délire poétique renvoie à la situation mal aisće du compositeur; à un doute profond par rapport à sa pratique. A cet égard Adorno obscrve que "l'expressionnisme est hostile à l'oeuvre".

Schönberg s'oriente ensuite vers le dodécaphonisme et le retour à l'usage des figures du contrepoint classique. Mais le musicien viennois se souvient des formes anciennes et Boulez fera remarquer que:

la série intervient chez Schönberg comme un plus petit commun dénominateur pour assurer l'unité sémantique de l'ocuvre; mais que les ćléments du langage ainsi obtenus sont organisés par une rhétorique préexistante, non sérielle.

Les formes anciennes sont une référence quasi divine et le rationalisme de la série lui est encore assujetti. On pourrait alors comparer Schßnberg avec Descartes lorsque ce dernier sépare le monde du raisonnement -qui agit sur le matériel- et la transcendance divine. Hugues Dufourt analyse ainsi la position du philosophe: Cassirer voit dans l'infinitisme cartésien une étape dans un processus d'émancipation qui s'achèvera avec Leibniz et Kant. L'opposition cartésienne entre la finitude de l'esprit humain et l'infinie puissance de Dieu constitue aux ycux de Cassirer un obstacle à l'avènement du rationalisme moderne... La véracité divine, chez Descartes, garantissait à la fois la valeur de nos raisonnements et l'existence du monde extérieur. L'idée du parfait et l'jdée de notre existence qui comprend l'idée du parfait ríclament Dieu comme cause. Cassirer voit dans cette disproportion l'indice d'une persistance médiévale. ${ }^{3}$

En ce qui concerne Schönberg, compositeur à la croisée des chemins, il s'agirait, si l'on tente le parallèle, de persistance romantique.

2 Theodor W. ADORNO, Philosophie de la non'elle musique, Gallimard, Paris, p.60.

3 Hughes DUFOURT, "Les fonctions paradigmatiques de la musique chez Leibniz", in Cahiers dil CIREMf No35-36, Tours, 1995, p.32-33. 
Webern va entrainer le langage schonbergien vers plus d`abstraction. Les références aux formes classiques - sonate, variations - deviennent chez lui de pius en plus symboliques et sa rationalisation le mene vers la notion de modèle, de forme absolue aux miroirs multiples. Il s'agit de sérier les paramètres musicaux (hauteurs, rythme, dynamique, registres).

Quand, lors de conférences, Webern présente le second mouvement de sa Symphonie, il en décrit la forme en concluant: il est impossible d'avoir plus de cohérence. Les Franco-flamands eux-mêmes ne sont pas parvenus à plus de colérence... Le mouvement en son entier constitue un double canon avec rétrograde.

Je citerai pour finir cette ancienne senterce latine:

SATOR
AREPO
TENET
OPERA
ROTAS

Au lendemain de la Seconde Guerre Mondiale un bouleversement profond se produit. $\mathrm{Ce}$ qui arrive en musique peut-il être comparé à l'introduction de la physique méciniste par Descartes dans le savoir de son temps? Il serait certainement tàntaisiste d'établir un parallèle tant les situations sont difficilement comparables.

Mais, il apparait un phénomène de table rase, et pour nombre de compositeurs après 1945, le langage doit se fonder sur d'autres bases que sur la syntaxe traditionnelle épuisėe. La nouvelle musique se reconnaît d'abord dans l'hẻritage webernien et étaye son discours par des références à la science.

Ainsi, lorsqu'il expose ses principes de composition, Stockhausen cite Helmholtz et son essai Comment passe le temps... est révélateur de la nouvelle pensée musicale:

\begin{abstract}
Dans la musique sérielle on tente d'ordonner les proportions de temps des éléments à l'aide de séries. L'organisation st́rielle s'appliquait tout d'abord à la portion des proportions de temps qui sont ressen. ties comme des hauteurs de sons. On a pris comme étant donné le sy'stème des douze sons par octave. Les douze sons étaient un nombre raisonnablement limité de grandeurs. Ces grandeurs perrnettaient de former une série organisée qui se caractériserait par divers rapponts - par la répartition des onze intervalles de grandeur à grandeur. ${ }^{4}$
\end{abstract}

La série abstraite devient ume série de proportions d'harmoniques (sur le modèle des partiels d'un son fondamental) et ce principe est appliqué à l'ensemble des paramètres, y compris aux tempi. Une telle extension du sćrialisme se rapproche d'une pensée algorithmique.

Le terme algorithme renvoie, dans le domaine musical, à P'ierre Barbaud qui, en 1950, est le premier qui cherchè à "introduire la pensée mathèmatique et les méthodes qui en tieconlent dans la composition musicale". Il imagine les programmes Algol et précise sa pensée dans des textes aux titres péremptoires: "La composition automatique", "La .1/usique, discipline scientifique".

On pense alors à Xenakis, à ses musiques aux litres significatifs, tel que Pithoprakta (Actions par probabilités), qui énoncent le mode de composition issu des mathématiques.

+ Karlheinz STOCKIIAUSEN, Comment passe le temps..., traduction française in Contrechamps $N^{\circ} 9$. Lausanne, 1988, p.29. 
Les nuages sonores de l'orchestre sont réglés selon la théorie cinétique des gaz et les éléments sont distribués suivant la loi de Poisson. Pour le compositeur, l'ère des arts scientifiques et philosophiques est commencée. Désormais le musicien devra être un fabricant de thèses philosophiques et d'architectures globales, de combinaisons de structures et de matrices sonores.

Suivant ses principes Xenakis mele architecture et musique utilisant pour le Pavillon Philips de l'Exposition de Bruxelles (1958) des éléments calculés pour les pentes des glissandi des cordes à la fin de Metastaseis pour orchestre.

Notons que cinq cents ans plus tôt Guillaume Dufay avait procédé de manière inverse en associant architecture et musique dans son motet "Nuper rosarum flores". Les proportions numériques de la pièce reproduisent celles de la coupole de Brunelleschi pour Santa Maria del Fiore à Florence.

En parallèle, Boulez va aflirmer: Cette pensée sérielle peut enlin sortir du chiflire douze, où elle était restée enfermée longtemps, ct pour cause; puisque ce sont justement les douze sons, c'est-à-dire le chromatisme, qui ont permis le passage de la structure tonale de plus en plus faible, à la structure sérielle. En définitive, ce ne sont pas les douze sons qui ont la plus grande importance; bien plutôt, la conception sérielle; soit la notion d'un univers sonore - propre à chaque oeuvre- se fondant sur un phénomène indiflërencié jusqu'au monent où l'on choisit sa séric: il devient alors unique et essentiel. ${ }^{5}$

Il en découle, toujours selon Boulez que: les recherches importantes sur les qualites intrinsèques du son sont à entreprendre; les appareils perfectionnés, maniables, nécessaires à la composition d'oeuvres telles, ne sont pas encore construits, écrivait-il en 1957. Néanmoins, ces vues ne sont pas utopiques jusqu'à pouvoir les ignorer; il est mẻme probable que l'intérèt croissant suscité par l'épiphanie d'un monde sonore inusité, inoü, ne fera qu'accélérer les solutions. Souhaitons d'en être les premiers artisans, modestement. ${ }^{6}$

Dans les annẻes soixante-dix finissantes le travail sur la synthèse sonore a déjà commencé, dans un premier temps issu de la réflexion sur la décomposition périodique du son (d'après la loi de Fourier).

Une grande part de la synthèse additive est adossée à cette technique qui eut d'importantes répercussions esthétiques, ne serait-ce qu'à travers la problématique dite du spectre. ${ }^{7}$ Dans une démarche issue du sérialisme Stockhausen avait déjà eu cette intuition avec les groupes de formants dans Gruppen. Avec la tendance, dite spectrale des compositeurs francais Grisey et Murail, on obtient, dans une réalisation purement instrumentale, la simulation de textures de spectres reconstitués de cloches ou de trombone. L'analyse spectrographique a permis à ces musiciens de mettre en oeuvre des processus compositionnels dans lesquels un spectre peut, par exemple, se transformer progressivement en un autre. Avec les nouvelles générations de studios informatisés, la synthèse sonore peut s'ėpanouir; le son va trouver ses microscopes géants.

Après les périodes sérielle, électroacoustique, électronique, l'ordinateur, dont l'importance dans la composition avait été pressentie dès les années soixante par Xenakis, fait son entrée. Au plus haut niveau de la technologie les équipements de l'IRCAM ont permis de réduire l'écart entre les chercheurs et les compositeurs.

5 Pierre BOULEZ, in Releves d'appreni, Editions du Seuil, Paris, 1966, p.228.

6 Ibidem, p.23!.

7 François NICOLAS, "Pour une intellectual its musicale", Inharmoniques N8/9, 1RCAM, Paris, p. 213. 
Les possibilités offertes sont désormais immenses, mais l'objet technologique ne peut cacher les enjeux fondamentaux de la composition.

Ce qui compte, écrit Jean-Claude Risset, c'est moins le matériel, la structure, les circuits, la machine, que le logiciel, la fonction, les programmes, le savoir-faire - l'investissenent intellectuel de la pratique, de l'expérience et de la recherche. ${ }^{8}$

Si nous considérons alors le cas du système de transformation en temps réel, comme exemple de l'incidence de l'ordinateur dans le processus musical, Répons de Pierre Boulez utilise des transformations en temps réel qui permettent de spatialiser mais surtout d'amplifier un ensemble de chambre à une dimension quasi symplıonique. Mais le temps réel concerne concerne l'interprétation plus que la composition: nombre de transformations compositionnelles sont incompatibles avec le temps rél. ${ }^{9}$

L'informatique musicale étend le domaine de la synthèse sonore:

On peut étendre la démarche compositionnelle jusqu'à la composition des sons euxmèmes, envisager toutes les implications potentielles de cette situation nouvelle.

Jean-Claude Risset l'a expérimenté dans ses oeuvres; on peut évoquer l'exemple du son qui monte et descend en même temps. C'est un artefact obtenu grâce à l'informatique qui permet à un son jusque là impossible d'exister. C'est un avatar du rapport entre réel et rationnel.

On pourrait brièvement conclure de toutes ces expériences, fruit de la formidable accélération de ces dernières décennies, en envisageant le rationnel dans l'acte compositionnel dans un champ de réflexion plus large. Citons encore Dufourt:

La musique ne se borne pas à un calcul, à une manipulation extérieure de symboles. Elle implique l'échange de l'avenir et du passe. Ainsi progresse-t-elle d'une dètermination à une autre, non par l'application d'une règle logique assignable, mais en reforinulant ses expressions, en redéfinissant les liaisons concrètes et abstraites, en réaménageant les rapports de l'intuition et du symbolisme qui la soutient... Le travail de composition procède par approches et corrections successives, par ajustements progressifs du symbole et de l'intuition, du formel et du concret. ${ }^{10}$

Dans l'évolution de la composition, le vingtième siècle aura tenté de montrer que la musique est art et science. On peut alors citer Boulez en conclusion:

On a maintes fois répété: la musique est une science autant qu'un art; qui saura londre ces deux entités au même creuset sinon l'Imagination, cette reine des facultés. "l

8 Jean-Claude RISSET, "Musique, recherche, theorie, espace, chros", Inharmoniques No3/9, p.284-285.

9 Ibidem, p.292.

10 Jlugues DUFOURT, A/nsique, ponoir, écriture, Ch. Bourgois, Paris, 1991, p.312.

11 Pierre BOULEZ, Penser la musique anjourd'hui, Denosl-Gonthier, Paris, 1964. 